\title{
Trainability of cold induced vasodilatation in fingers and toes
}

\author{
Hein A. M. Daanen · Jens Koedam • \\ Stephen S. Cheung
}

Received: 10 April 2011/Accepted: 31 October 2011/Published online: 12 November 2011

(C) The Author(s) 2011. This article is published with open access at Springerlink.com

\begin{abstract}
Subjects that repeatedly have to expose the extremities to cold may benefit from a high peripheral temperature to maintain dexterity and tissue integrity. Therefore, we investigated if repeated immersions of a hand and a foot in cold water resulted in increased skin temperatures. Nine male and seven female subjects (mean 20.4; SD 2.2 years) immersed their right (trained) hand and foot simultaneously in $8^{\circ} \mathrm{C}$ water, 30 min daily for 15 days. During the pre and post-test (days 1 and 15 , respectively) the left (untrained) hand and foot were immersed as well. Pain, tactile sensitivity and skin temperatures were measured every day. Mean (SD) toe temperature of the trained foot increased from $9.49^{\circ} \mathrm{C}$ $(0.89)$ to $10.03^{\circ} \mathrm{C}(1.38)(p<0.05)$. The trained hand, however, showed a drop in mean finger temperature from $9.28^{\circ} \mathrm{C}(0.54)$ to $8.91{ }^{\circ} \mathrm{C}(0.44)(p<0.001)$ and the number of cold induced vasodilation (CIVD) reactions decreased from $52 \%$ during the first test to $24 \%$ during the last test. No significant differences occurred in the untrained extremities. Pain diminished over time and tactile sensitivity decreased with skin temperature. The combination of less CIVD responses in the fingers after training, reduced finger skin temperatures in subjects that did show CIVD and the reduced
\end{abstract}

Communicated by George Havenith.

H. A. M. Daanen ( $₫)$

Department of Human Performance, TNO, PO Box 23,

3769 ZG Soesterberg, The Netherlands

e-mail: hein.daanen@tno.nl

H. A. M. Daanen · J. Koedam

Research Group MOVE, Faculty of Human Movement Sciences, VU University, Amsterdam, The Netherlands

S. S. Cheung

Department of Kinesiology, Brock University,

St. Catharines, Canada pain and tactile sensitivity over time may lead to an increased risk for finger cold injuries. It is concluded that repeated cold exposure of the fingers does not lead to favorable adaptations, but may instead increase the injury risk.

Keywords Temperature - Thermoregulation · Cold · Extremities · Vasodilation · Trainability

\section{Introduction}

When humans are exposed to cold, information from skin temperature sensors and blood temperature sensors in the hypothalamus activates the sympathetic nervous system, leading to skin vasoconstriction and reduced heat loss. The downside of this mechanism is that the blood flow in the fingers and toes may become so low that tissue integrity is at stake (Wilson and Goldman 1970). Fortunately, at extremity skin temperatures below about $15^{\circ} \mathrm{C}$ a sudden periodic vasodilatation is often observed, first described by Lewis (1930). The most likely mechanism for this vasodilatation is the muscular wall of the arterio-venous anastomoses in the fingers and toes which cannot maintain vascular tone when cooled (Daanen 2003). The opening of these vessels lead to increased blood flow and, indirectly, increased local skin temperatures. Regardless of the underlying mechanisms, this cold induced vasodilatation (CIVD) response is often considered as a protective mechanism against local cold injuries (Daanen 2003; Wilson and Goldman 1970). Indeed, it has been shown that subjects with a reduced CIVD response have a higher risk of frostbite (Daanen and Van Der Struijs 2005). Therefore, it may be desirable for subjects working in the cold to have a fast CIVD response of high magnitude, leading to research into how to manipulate and possibly enhance CIVD. 
The idea of using repeated local cold exposure as a training stimulus for increased CIVD comes from population cross-sectional studies. These studies showed that natives of circumpolar environments (e.g., Inuits and Sami) exhibit a higher mean finger temperature compared to people who live in a more temperate climate when exposed to cold (Krog et al. 1960; Miller and Irving 1962). An enhanced CIVD reaction was also observed in fishermen compared to a control group less regularly exposed to cold (Krog et al. 1960). Nelms and Soper (1962) found that fish filleters, who are exposed to local cold only, also showed a significant earlier CIVD reaction compared to the control group. However, the possibility of self-selection for these particular occupations should not be excluded. The effects of repeated cold exposure on CIVD in field studies show ambiguous results. Some studies found a lowered mean finger temperature after a 2-week Arctic expedition, which may indicate a higher risk for cold injuries (Livingstone 1976). On the other hand, Purkayastha et al. (1992) observed higher finger temperatures in Indian soldiers after a 7-week expedition to the Arctic environment.

In all reported lab studies on trainability, only either the hand or the foot was exposed to cold. Three studies were identified in which the foot was trained (Reynolds et al. 2007; Savourey 1996; Yoshimura 1960). The study of Reynolds et al. (2007) observed no differences, the other two studies observed higher mean foot temperatures after repeated immersion. For hand immersion, most studies showed no training effect (Eagen 1966; Geurts et al. 2006a; Glaser and Whittow 1957; O'Brien 2005), three studies showed a negative training effect (Daanen et al. 2007; Geurts et al. 2005a; Mekjavic et al. 2008) and only two studies showed positive effects (Adams and Smith 1962; Geurts et al. 2006b). In summary, it seems that training of the foot is more successful than training of the hands or fingers. CIVD of the fingers is generally much more pronounced than CIVD of the toes (Cheung and Mekjavic 2007; Van Der Struijs et al. 2008) and there may be more scope for trainability in the toes than in the fingers.

Therefore, a study was performed in which the CIVD trainability was investigated for fingers and toes. This was done by tracking thermal responses of the fingers and toes over 15 days of repeated local immersion in $8^{\circ} \mathrm{C}$ water. In line with the general picture from the existing literature, we hypothesized that toe CIVD would show more improvement than finger CIVD following repeated immersions.

\section{Methods}

\section{Subjects}

Eighteen students volunteered for participation in this study. During the first day, two subjects indicated that they could not tolerate the cold water during immersion of the hands and feet and were withdrawn. The remaining 16 subjects, nine males and seven females, participated until the end. The subjects had a mean (SD) age of 20.4 (2.2) years, mean (SD) height of $177.7(1.1) \mathrm{cm}$, and mean (SD) body mass of $71.5(12.4) \mathrm{kg}$. One subject was left-handed, 15 were right-handed and all were non-smokers.

The subjects followed a protocol approved by the Human Ethics Committee of the TNO. All subjects were informed about the potential risks and discomforts of the study and gave their written informed consent to participate. All subjects were medically screened for cardiovascular diseases and Raynauds syndrome.

\section{Protocol}

After entering the room (ambient temperature $20-24^{\circ} \mathrm{C}$, relative humidity $30-70 \%$ ), the subject sat down on a chair. The subjects wore shoes, socks, trousers or shorts and a shirt (one single layer of clothing). For the first $10 \mathrm{~min}$, the subjects rested while the measurement equipment was attached to the body by the researcher. Thermistors at the fingers were taped just lateral of the nail bed, since the reproducibility of skin temperature is better in the nail bed (O'Brien 2005). The thermistors for the toes were taped at the 'toe pad' since Reynolds et al. (2007) found a highly variable CIVD response at the nailbed of the toes. Therefore we chose for the toe pad, which is the common position in most previous research, so that comparison to previous studies is enabled (e.g. Van Der Struijs et al. 2008). Five minutes prior to immersion of the hand and foot in cold water, the subjects immersed their hand and foot in water of $34-36^{\circ} \mathrm{C}$ to create similar starting conditions for every subject. The hand was positioned at the level of the heart, while the foot was placed in a water tank located on the floor. The hand as well as the foot made no contact with the bottom of the water tanks, because of a water-permeable mesh (3mesh, Muller Textil GmbH, Wiehl, Germany) that was lying at the bottom of the water tanks.

After $5 \mathrm{~min}$ in warm water the subject put the right hand (up to the ulnar and radial styloids) and foot (up to the malleolus) for 30 min into cold stirred tap water of $8^{\circ} \mathrm{C}$. The immersion baths were placed next to the warm water baths. The hand and the foot were immersed simultaneously. Every $5 \mathrm{~min}$, starting at minute five, tactile sensitivity of the tip of the index finger was tested with Semmes-Weinstein monofilaments. Directly after the Semmes-Weinstein test, the subjects used the nonimmersed hand to fill in a pain score for their hand as well as foot. After $30 \mathrm{~min}$ of cold immersion, the hand and foot were taken out of the water tanks and patted dry with a towel. The temperature measurements ended 5 min later. 
This protocol was repeated over 15 days, 5 days per week. Day 1 was the pre-test and day 15 the post-test session. There were 2 days between the pre-test and the second immersion. Between day 14 and the post-test, the subjects had one day off. The left, non-acclimatized hand and foot were immersed at day 1 (pre-test) and 15 (post-test) as well, before or after (counterbalanced) the immersion of the right hand and foot.

\section{Materials}

The hand immersion bath had a dimension of $49.5(l) \times$ $29(w) \times 13(h) \mathrm{cm}$, and the foot water bath had a dimension of $46(l) \times 36(w) \times 22.5(h) \mathrm{cm}$. To minimize the heat loss or heat gain of the water in the baths, they were insulated with polystyrene. The temperature was controlled within $1^{\circ} \mathrm{C}$ using a thermostat bath (TLC 15, PM Tamson Instruments, Bleiswijk, The Netherlands).

Finger and toe temperatures were continuously monitored using thermistors (type P-8432, ICBT, Tokyo, Japan) attached to the skin by one layer of Leukoplast tape (BSN medical \& GmbH \& Co.KG, D-22771, Hamburg, Germany) and connected to a Mobi8 data acquisition system (TMS International BV, Oldenzaal, The Netherlands). The temperature of the fingers and toes was sampled every second. The lowest value over the $30 \mathrm{~min}$ immersion interval was defined as the minimum temperature $\left(T_{\min }\right)$. The mean ( $\left.T_{\text {mean }}\right)$ and maximum $\left(T_{\max }\right)$ temperatures were calculated over the 5- to 30-min interval. CIVD reactions were defined as a continuous rise of at least $1^{\circ} \mathrm{C}$. To exclude minor fluctuations, we averaged the values over a period $20 \mathrm{~s}$ before and $20 \mathrm{~s}$ after the measurement for all temperatures. When the rise was $<1^{\circ} \mathrm{C}$, the response was counted as 'No CIVD', when it was $1^{\circ} \mathrm{C}$ or more it was counted as a CIVD response. The onset time is the time in seconds from start of the immersion until the start of a continuous increase of temperature of at least $1^{\circ} \mathrm{C} . T_{\text {peak }}$ is the temperature at the peak of the first CIVD wave. The CIVD analysis was completely automated to exclude human subjectivity.

Pain was assessed every 5 min using a $0-10$ visual analog scale (VAS) Numeric Pain Distress scale. Tactile sensitivity at the tip of the index finger was assessed using Semmes-Weinstein monofilaments (Bell-Krotoski and Tomancik 1987). The subjects turned the hand under water every $5 \mathrm{~min}$ for about $10 \mathrm{~s}$ to enable determination of tactile sensitivity.

\section{Statistical analysis}

Since most studies have limited statistical power, we included 16 subjects in the study. Based on finger pad temperatures in the study of O'Brien (2005), differences in onset time of $25 \mathrm{~s}$ and differences in peak temperature of $1.5^{\circ} \mathrm{C}$ can be detected. We measured all fingers and toes because prevalence of a CIVD in a single finger or toe shows considerable variability (Cheung and Mekjavic 2007; Mekjavic et al. 2008; Reynolds et al. 2007).

Statistical analyses were performed using Statistica (Statsoft 2008). Data for skin temperature, pain and tactile sensitivity were compared using a two-way (condition $\times$ time) factorial analysis of variance (ANOVA), with finger and toe temperatures statistically treated as independent. CIVD parameters between pre- and post-test were identified using a paired $t$ test. Differences in the number of CIVD reactions between pre- and post-test were analyzed non-parametrically. The results are expressed as means (SD), and significance was accepted as $p<0.05$.

\section{Results}

The finger and toe skin temperatures averaged over all fingers and toes during the days of immersion are shown in Fig. 1. Finger skin temperature essentially remains constant; toe skin temperature shows an increase. During the last 2 days a drop in toe and finger skin temperature was noticed.

Cold induced vasodilation parameters of the pre- and post-test are shown in Table 1. Due to technical problems with the attachment of the sensors to the skin, there were several drop-outs randomly divided over the subjects during the first day. While the untrained extremities did not change in temperature after 15 days of immersion, the trained extremities did change. The number of CIVD reactions with at least one wave dropped significantly in trained fingers after 15 days of cold water immersion from 52 to $24 \%(p<0.01)$ while it remained unchanged in the untrained fingers (39 vs. $46 \%$ ). The onset times of CIVD

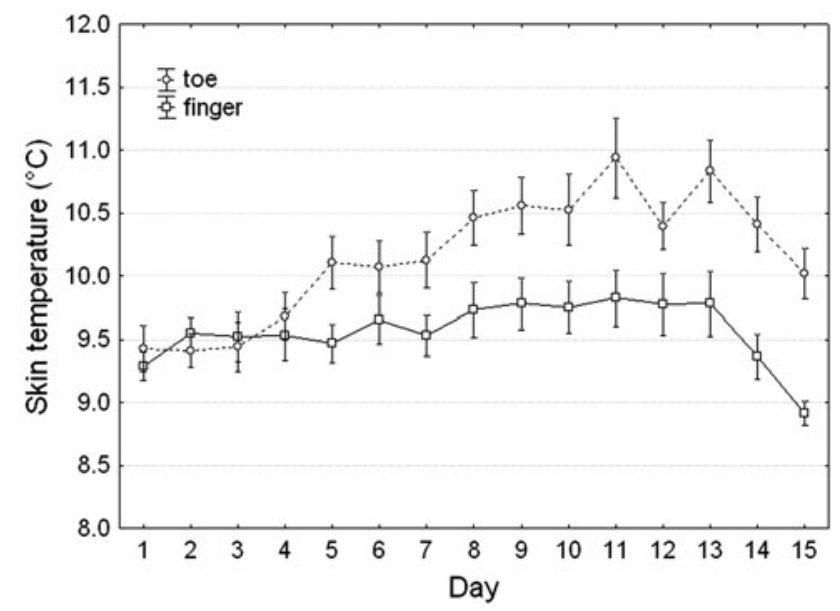

Fig. 1 Finger and toe skin temperatures $\left({ }^{\circ} \mathrm{C}\right)$ averaged over minutes 5-30 and over all digits during immersion in $8^{\circ} \mathrm{C}$ water for 15 consecutive days. Vertical bars denote the standard error of the mean. All values are in ${ }^{\circ} \mathrm{C}$ 
Table 1 Comparison between pre- and post-test for CIVD parameters

\begin{tabular}{|c|c|c|c|c|c|c|c|c|}
\hline & \multicolumn{4}{|l|}{ Pre-test } & \multicolumn{4}{|l|}{ Post-test } \\
\hline & \multicolumn{2}{|l|}{ Fingers } & \multicolumn{2}{|l|}{ Toes } & \multicolumn{2}{|l|}{ Fingers } & \multicolumn{2}{|l|}{ Toes } \\
\hline & Trained & Untrained & Trained & Untrained & Trained & Untrained & Trained & Untrained \\
\hline$N$ & 73 & 64 & 63 & 68 & 79 & 80 & 79 & 79 \\
\hline \#CIVD & $38=52 \%$ & $25=39 \%$ & $28=44 \%$ & $30=44 \%$ & $19=24 \%^{\Delta}$ & $37=46 \%$ & $23=29 \%$ & $30=38 \%$ \\
\hline Onset time (s) & $676(462)$ & $629(456)$ & 766 (292) & 763 (349) & $716(407)$ & $629(471)$ & 735 (338) & 741 (419) \\
\hline$T_{\text {peak }}\left({ }^{\circ} \mathrm{C}\right)$ & $10.54(0.69)$ & $11.88(2.07)$ & $11.17(0.99)$ & $12.64(1.81)$ & $10.27(0.79)$ & 11.48 (1.90) & $11.85(2.05)$ & $12.60(2.35)$ \\
\hline$T_{\text {mean }}\left({ }^{\circ} \mathrm{C}\right)$ & $9.28(0.54)$ & $9.41(1.03)$ & $9.49(0.89)$ & $9.73(1.04)$ & $8.91(0.44)^{*}$ & 9.55 (1.10) & $10.03(1.38)^{\dagger}$ & $9.96(1.23)$ \\
\hline$T_{\min }\left({ }^{\circ} \mathrm{C}\right)$ & $8.69(0.32)$ & $8.69(0.41)$ & $8.81(0.72)$ & $8.88(0.70)$ & $8.48(0.24)^{*}$ & $8.72(0.45)$ & $9.15(0.89)^{\dagger}$ & $8.97(0.78)$ \\
\hline$T_{\max }\left({ }^{\circ} \mathrm{C}\right)$ & $10.21(0.91)$ & 10.49 (1.89) & $10.85(1.23)$ & $11.54(1.76)$ & $9.63(0.86)^{*}$ & $10.72(2.13)$ & $11.68(2.16)^{\Delta}$ & $11.92(2.23)$ \\
\hline
\end{tabular}

$N$ is the number of experimental datasets. The maximum is 16 subjects $\times 5$ toes/fingers $=80$. Mainly in the pre-test, several measurements had to be disregarded since the sensors became detached from the skin. \#CIVD reactions stand for the number of experimental datasets with at least one CIVD reaction. Values are mean (SD)

* Significantly different from pre-test values $(p<0.001)$

${ }^{\Delta}$ Significantly different from pre-test values $(p<0.01)$

$\dagger$ Significantly different from pre-test values $(p<0.05)$

were widely spread and did not display significant differences; neither did the peak of the CIVD wave. While the trained fingers showed a significant decrease in temperature (for $T_{\text {mean }}, T_{\min }$ and $T_{\max }, p<0.001$ ) during the posttest, the trained toes showed an increase in temperature during the post-test $\left(T_{\text {mean }}\right.$ and $T_{\min }, p<0.05 ; T_{\max }$, $p<0.01$,). Finger skin temperatures were significantly lower for the thumb $(p<0.05)$, index finger $(p<0.05)$, middle finger $(p<0.05)$, ring finger $(p<0.01)$ and little finger $(p<0.001)$ during day 15 as compared to day 1 . Toe temperatures were higher for the middle $(p<0.05)$, fourth $(p<0.01)$ and little toe $(p<0.05)$ and not significantly different between day 1 and day 15 for the big toe and the index toe.

Pain in the trained hand and foot decreased over time, as can be clearly seen in Fig. 2. This graph displays the average pain experienced during cold water immersion each of the 15 days. Pain in the hand dropped from 4.5 (1.8) at day 1 to $2.9(1.9)$ at day $15(p<0.001)$. Pain in the foot dropped from $4.6(1.7)$ to 2.3 (1.8) $(p<0.001)$. After day 4 , there was no further significant drop in pain.

Pain was significantly less during post-test compared to the pre-test (Table 2). This is true for hand as well as foot and trained side as well as the untrained side. Because the scores of the trained and untrained hand at day 1 were equal (no significant change between these scores), the comparison between the scores at day 15 show significantly less pain in the trained hand, compared to the untrained hand at day 15 . The same is true for foot, but a note has to be made here, because pain in the trained foot at day 1 was already significantly lower compared to the pain in the untrained foot $(p<0.05)$.

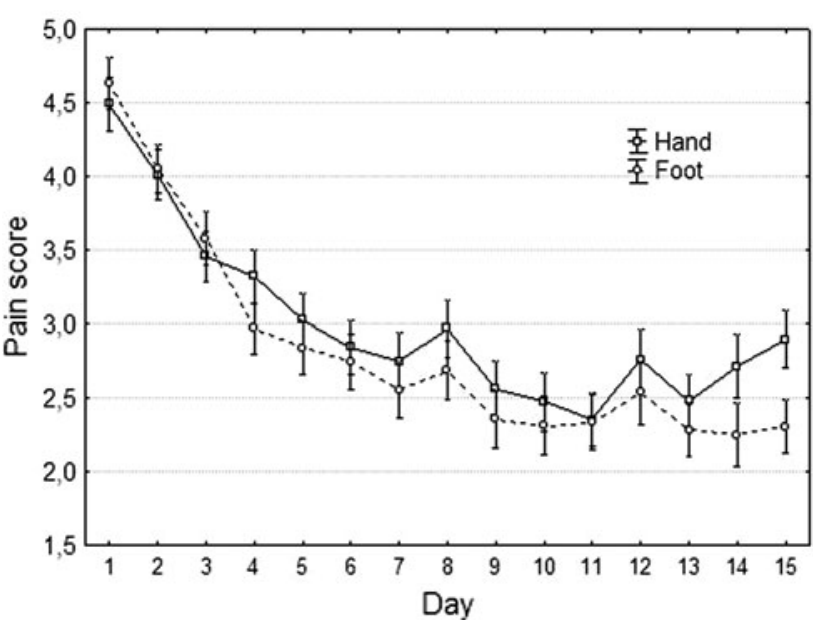

Fig. 2 Average pain score and standard error of the mean of the trained hand and foot during immersion over 15 days. Note that at day 1 and 15 the immersion sequence of the extremities was counterbalanced

Tactile sensitivity of the index finger did not change significantly over 15 days, but was related to the skin temperature of the index finger. Figure 3 shows the relationship between index finger skin temperature and tactile sensitivity. For a mean (SD) skin temperature of $8.78^{\circ} \mathrm{C}$ (0.35) only a thick filament $(4.08 \mathrm{~g})$ could be detected, whereas for an index finger skin temperature of $10.35^{\circ} \mathrm{C}$ (1.83) even thin filaments of $2.44 \mathrm{~g}$ could be detected.

\section{Discussion}

The main objective of this study was to investigate the effect of repeated local cold exposure on thermal responses 
Table 2 Pre- (day 1) and post-test (day 15) pain scores (SD)

\begin{tabular}{lll}
\hline Pain & Day 1 & Day 15 \\
\hline Trained hand & $4.5(1.8)$ & $2.9(1.9)^{*}$ \\
Untrained hand & $4.8(1.8)$ & $3.9(2.2)^{*}$ \\
Trained foot & $4.6(1.7)$ & $2.3(1.8)^{*}$ \\
Untrained foot & $5.1(1.7)$ & $4.2(2.1)^{*}$ \\
\hline
\end{tabular}

* Significantly different from day 1 values $(p<0.001)$

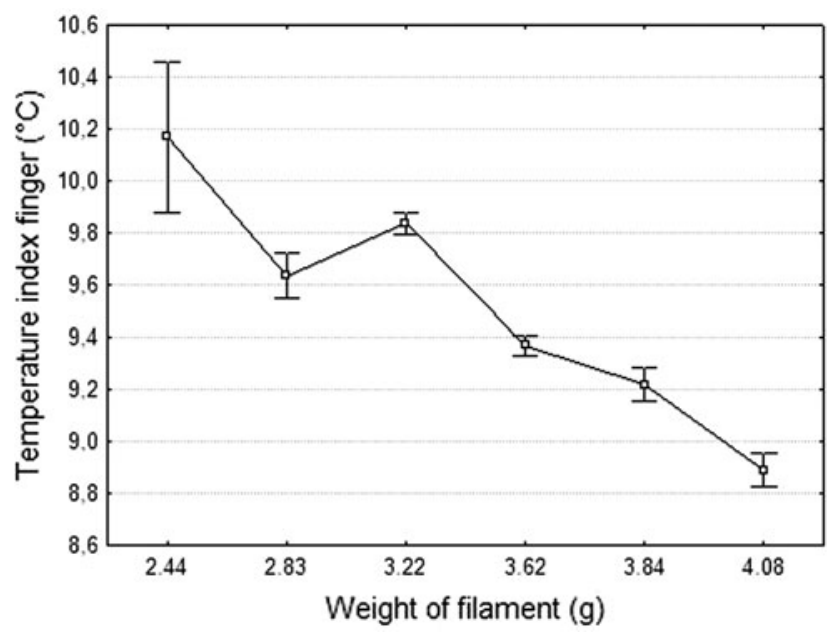

Fig. 3 Relation between index finger skin temperature and tactile sensitivity. Tactile sensitivity is expressed as the weight of the Semmes-Weinstein filament. Data for this figure included all measurements over the 15 days of temperature and sensitivity (trained as well as untrained index finger). Vertical bars denote the standard error of the mean

in both the fingers and toes. Furthermore, we looked at the influence of local cold acclimation to pain and tactile sensitivity. Fifteen days of immersing the right hand and foot simultaneously in cold water for 30 min produced somewhat contradictory responses in the fingers versus the toes. Namely, the incidences of CIVD in toes pre- and postacclimation were not different, from $44 \%$ of the possible events pre-acclimation to $29 \%$ post-acclimation. However, the $T_{\text {mean }}, T_{\min }$, and $T_{\max }$ increased post-acclimation, especially in the third, fourth, and fifth toes; although significant, the magnitude of the changes in our study is small, at $<1^{\circ} \mathrm{C}$ on average. Our data, combined with the positive training effect for toes observed by Yoshimura (1960) and Savourey (1996), seem to suggest that toe blood flow is trainable, albeit modestly. To date, only Reynolds et al. (2007) observed no changes in a similar study.

In contrast, the fingers proved unresponsive to repeated cold exposures, and indeed the thermal response may have degraded. The number of CIVD reactions in the fingers dropped significantly from 54 to $24 \%$, post-acclimation, as did the finger skin temperature parameters. Less subjects showed CIVD post-acclimation and the subjects that still had CIVD showed it to a lesser extent. Results in the literature are conflicting, but the majority of studies observe no effect or a detrimental effect of repeated cold exposure to finger skin temperature. The decrease of CIVD reactions and temperature in fingers are in line with previous findings by Mekjavic et al. (2008), who found similar results of these parameters after 13 days of right hand immersion in water of $8^{\circ} \mathrm{C}$ for $30 \mathrm{~min}$ and Geurts et al. (2005b), who reported a drop of mean finger skin temperature after investigating the CIVD response with ten subjects in similar conditions as used in our study. Most studies observed no effects (Eagen 1966; Geurts et al. 2006a; Glaser and Whittow 1957; O'Brien 2005), while only two studies observed positive effects (Adams and Smith 1962; Geurts et al. 2006b). Therefore, the risk for cold injuries in the fingers may be elevated in individuals acclimated to cold. This is further supported by a decrease in pain sensations with both hand and feet cooling after acclimation.

The decrease in finger skin temperature due to training was mainly observed during the last 2 days. This drop in finger and toe temperature during the last 2 days (Fig. 1) may be related to the ambient temperature outside of the building that was about $16^{\circ} \mathrm{C}$ during days 14 and 15 and $18-22^{\circ} \mathrm{C}$ in the preceding days (meteorological data from Schiphol airport that is in close vicinity to the measurement location). The lower ambient temperature may have caused a reduction in the thermal state of the body prior to the experiment. Controlling the temperature of the experimental room and immersing the extremities in warm water $\left(35^{\circ} \mathrm{C}\right)$ for $5 \mathrm{~min}$ prior to the cold water immersion with the intention to create a stable thermal condition for the subjects may have been insufficient to eliminate the effect of the external ambient temperature on the thermal status of the body. On the other hand, it may simply be that the changes during the last 2 days reflect real changes due to cold training that may only take effect following prolonged and repeated exposures. The ambient temperature was identical at day 1 and day $15\left(16^{\circ} \mathrm{C}\right)$; therefore, a fair comparison can be made between the pre- and post-test.

The decrease in finger skin temperature and increase in toe skin temperature during the CIVD training period of 15 days may be partly attributed to anatomical differences. The feet have a smaller surface area to volume ratio than the hands (68 vs. $104 \mathrm{~m}^{-1}$ for men (Tikuisis et al. 2001), which leads to slower cooling of the feet as compared to the hands. This may be the reason that CIVD is less pronounced in the feet than in the hands (Van Der Struijs et al. 2008). Therefore, it may be more effective to enhance vasoconstrictory tone and the sensitivity of local blood vessels for cold in the fingers as opposed to the toes to maintain the overall thermal status of the body. These changes may have taken place over the 15 days of 
investigation. Another explanation may be that since the toes are more prone to cold injuries than fingers (Daanen and Van Der Struijs 2005), it is more important to maintain tissue integrity in the toes and thus enhance local blood flow there.

The observed changes in finger and toe temperatures after 15 days of cold water immersion in our study are not likely to be of central origin. When sympathetic outflow would have increased, both fingers and toes would have shown vasoconstriction. Therefore, a peripheral mechanism should be held responsible for the observed changes, such as an increased sensitivity to cold at the fingers leading to vasoconstriction and a decreased sensitivity to cold at the toes leading to a reduction in vasoconstrictory tone.

Pain during immersion decreased significantly during the 15 days of local cold exposure. This is true for both feet and hands and for the trained and untrained side. Figure 2 clearly shows that the greatest decline in pain occurs in the first few days, independently of possible adaptations in skin temperature. The decrease of pain in time corresponds to previous observations (Geurts et al. 2006a; Sawada et al. 2000). The relative increase in pain during days 8 and 12 may be explained by the fact that these measurement days were relatively often on Mondays, and the 2-day weekend break from cold exposure may have reduced pain resistance. Because pain can be seen as a warning signal of the body for extreme cold, some researchers claim that a decrease in finger temperature, in combination with a decrease in pain due to training, lead to a habituation effect (Geurts et al. 2006a; Sawada et al. 2000). When people experience less pain after a few days of repeated cold exposure it is not expected that they adjust their behavior to their real thermal status, which increases the risk of cold injuries.

Previous studies reported that tactile sensitivity was unaffected when measured before and after repeated cold exposure (Geurts et al. 2006a, b). Unlike those studies, tactile sensitivity in this study was measured while the hand remained immersed in cold water, such that no rewarming due to removal from the cold exposure occurred. Our results clearly show that a lower skin temperature leads to a decreased tactile sensitivity. This is in line with a deterioration of tactile sensitivity as a result of decreased skin temperature found by Gescheider et al. (1997), Green et al. (1979) and Daanen (2009).

For the untrained extremities no significant differences were detected in our study, so it can be concluded that the observed differences in finger and toe skin temperature must reflect local effects of repeated immersion. If there would have been a change in central drive due to repeated immersion, changes in both the trained and untrained extremities should have been observed. Sympathetic activity is the central drive that is generally considered to play a major role in the control of CIVD (Daanen 2003). The sympathetic nerves innervate the strong muscular wall of the arterio-venous anastomoses that are abundantly present at CIVD locations. Sympathetic activation thus leads to vasoconstriction and a reduced CIVD response (Daanen 2003). The absence of any change in the nontrained fingers and toes indicate that the sympathetic activity is probably unaltered due to training.

In summary, sixteen subjects simultaneously exposed a hand and foot to $8^{\circ} \mathrm{C}$ water for $30 \mathrm{~min}$ daily for a period of 15 consecutive days excluding weekends. The toe temperatures showed a small increase after 15 days, while the finger temperatures dropped, as well as the number of CIVD responses in the finger. Therefore, the risk for cold injuries may increase in trained fingers, also because pain sensation gradually decreased so that the warning system becomes less alert. We, therefore, do not recommend repeated cold water immersion of the hands as a method for preventing cold injuries.

Acknowledgments The authors wish to thank the subjects for their perseverance and Dr. Joe Layden for the critical review of the manuscript.

Open Access This article is distributed under the terms of the Creative Commons Attribution Noncommercial License which permits any noncommercial use, distribution, and reproduction in any medium, provided the original author(s) and source are credited.

\section{References}

Adams T, Smith RE (1962) Effect of chronic local cold exposure on finger temperature responses. J Appl Physiol 17:317-322

Bell-Krotoski J, Tomancik E (1987) The repeatability of testing with Semmes-Weinstein monofilaments. J Hand Surg 12:155-161

Cheung SS, Mekjavic IB (2007) Cold-induced vasodilatation is not homogenous or generalizable across the hand and feet. Eur $\mathbf{J}$ Appl Physiol 99:701-705

Daanen HAM (2003) Finger cold-induced vasodilation: a review. Eur J Appl Physiol 89:411-426

Daanen HAM (2009) Manual performance deterioration in the cold estimated using the wind chill equivalent temperature. Ind Health 47:262-270

Daanen HAM, Van Der Struijs NR (2005) Resistance index of frostbite as a predictor of cold injury in Arctic operations. Aviat Space Environ Med 76:1119-1122

Daanen HAM, Raymann RJEM, Stoop M (2007) Trainability of cold induced vasodilation. In: Proceedings of the 12th international conference on environmental ergonomics Piran, Slovenia, 19-24 Aug 2007, pp 317-319 (ISBN 978-961-90545-1-2)

Eagen CJ (1966) Method of estimating local tolerance to extreme cold-discussion. In: Yoshimura H, Weiner JS (eds) Human adaptability and its methodology proceedings of a symposium Kyoto Japan, Japan Society for the promotion of sciences, 12-14 Sept 1965, p 62

Gescheider GA, Thorpe JM, Goodarz J, Bolanowski SJ (1997) The effects of skin temperature on the detection and discrimination of tactile stimulation. Somatosens Mot Res 14:181-188 
Geurts CL, Sleivert GG, Cheung SS (2005a) Effect of cold-induced vasodilatation in the index finger on temperature and contractile characteristics of the first dorsal interosseus muscle during coldwater immersion. Eur J Appl Physiol 93:524-529

Geurts CLM, Sleivert GG, Cheung SS (2005b) Local cold acclimation of the hand impairs thermal responses of the finger without improving hand neuromuscular function. Acta Physiol Scand 183:117-124

Geurts CL, Sleivert GG, Cheung SS (2006a) Local cold acclimation during exercise and its effect on neuromuscular function of the hand. Appl Physiol Nutr Metab 31:717-725

Geurts CLM, Sleivert GG, Cheung SS (2006b) Central and peripheral factors in thermal, neuromuscular, and perceptual adaptation of the hand to repeated cold exposures. Appl Physiol Nutr Metab 31:110-117

Glaser EM, Whittow GC (1957) Retention in a warm environment of adaptation to localized cooling. J Physiol 136:98-111

Green BG, Lederman SJ, Stevens JC (1979) The effect of skin temperature on the perception of roughness. Sens Processes 3:327-333

Krog J, Folkow B, Fox RH, Andersen KL (1960) Hand circulation in the cold of Lapps and North Norwegian fisherman. J Appl Physiol 15:654-658

Lewis T (1930) Observations upon the reactions of the vessels of the human skin to cold. Heart 15:177-208

Livingstone SD (1976) Changes in cold induced vasodilation during Arctic exercises. J Appl Physiol 40:455-457

Mekjavic IB, Dobnikar U, Kounalakis SN, Musizza B, Cheung SS (2008) The trainability and contralateral response of coldinduced vasodilatation in the fingers following repeated cold exposure. Eur J Appl Physiol 104:193-199

Miller LK, Irving L (1962) Local reactions to air cooling in an Eskimo population. J Appl Physiol 17:449-455
Nelms JD, Soper DJ (1962) Cold vasodilatation and cold acclimatization in the hands of British fish filleters. J Appl Physiol 17:444-448

O'Brien C (2005) Reproducibility of the cold-induced vasodilation response in the human finger. J Appl Physiol 98:1334-1340

Purkayastha SS, Selvamurthy W, Ilavazhagan G (1992) Peripheral vascular response to local cold stress of tropical men during sojourn in the Arctic cold region. Jpn J Physiol 42:877-889

Reynolds LF, Mekjavic IB, Cheung SS (2007) Cold-induced vasodilatation in the foot is not homogenous or trainable over repeated cold exposure. Eur J Appl Physiol 102:73-78

Savourey G (1996) Hypothermic general cold adaptation induced by local cold acclimation. Eur J Appl Physiol 73:237-244

Sawada SI, Araki S, Yokoyama K (2000) Changes in cold-induced vasodilatation, pain and cold sensation in fingers caused by repeated finger cooling in a cool environment. Ind Health 38:79-86

Statsoft I (2008) STATISTICA (data analysis software system), version 8.0. http://www.statsoft.com

Tikuisis P, Meunier P, Jubenville CF (2001) Human body surface area: measurement and prediction using three dimensional body scans. Eur J Appl Physiol 85:264-271

Van Der Struijs NR, van Es EM, Raymann RJEM, Daanen HAM (2008) Finger and toe temperatures on exposure to cold water and cold air. Aviat Space Environ Med 79:941-946

Wilson O, Goldman RF (1970) Role of air temperature and wind in the time necessary for a finger to freeze. J Appl Physiol 29:658-664

Yoshimura H (1960) Acclimatization to heat and cold. In: Essential problems in climatic physiology, Nankodo Ltd, Tokyo, pp 61-75 